\title{
An effective Neurofeedback training, with cortisol correlates, in a clinical case of anxiety*
}

\section{Efectividad de un entrenamiento asistido por Neurofeedback, con correlatos de cortisol, en un caso clínico de ansiedad}

DOI: $10.11144 / J a v e r i a n a . u p s y 15-5 . e n t c$ Received: 25 May 2016 | Accepted: 28 September 2016

\author{
Marta Aliño Costa ${ }^{a}$ \\ Universitat de València, España \\ MARIEN GADEA \\ Universitat de València, España \\ VANESA Hidalgo \\ Universitat de València, España \\ Víctor PÉrez \\ Universidad Autónoma de Barcelona, España \\ Julio SAnjuán \\ Universitat de València, España
}

aE-mail: marta.alino@uv.es

Para citar este artículo: Aliño, C. M., Gadea, M., Hidalgo, V., Pérez, V., \& Sanjuán, J. (2016). An effective neurofeedback training, with cortisol correlates, in a clinical case of anxiety. Universitas Psychologica, 15(5). http:// dx.doi.org/10.11144/Javeriana.upsy15-5.entc

Acknowledgements: This study was supported by the Spanish Ministry of Education (grant number FPU-AP2012-05836)

\begin{abstract}
Neurofeedback treatments have shown successful results in anxiety disorders. The effectiveness of a betal Neurofeedback protocol was tested in a longitudinal clinical case study. A participant suffering from an anxiety syndrome underwent 10 sessions of Neurofeedback, in a protocol consisting of uptraining the betal rhythm $(16-21 \mathrm{~Hz})$ while downtraining the theta $(4-8 \mathrm{~Hz})$ band. State anxiety and salivary cortisol levels were measured during each of the 10 sessions following a pre/post design. Initial and final examinations of anxiety symptoms and sustained attention performance were also implemented. The final evaluation revealed that levels of anxiety fell within a normative range and that sustained attention had improved. A t-test for related samples disclosed a significant improvement of beta1 amplitude across the sessions, without modifications in untrained bands. A significant inverse correlation between beta1 amplitude and salivary cortisol was detected, suggesting that brain activity could be considered a marker of anxiety. The validation of the beta1 Neurofeedback protocol was assessed according to independence, trainability and interpretability criteria. We demonstrate the effectiveness of a neurofeedback protocol on anxiety and sustained attention, the success of which may lie in the reestablishment of an optimal cortical arousal capable of inhibiting elevated amygdalar activity. Keywords

Neurofeedback; anxiety; cortisol; beta1 band; theta band.
\end{abstract}

\section{RESUMEN}

El Neurofeedback (NF) ha demostrado exitosos resultados en trastornos de ansiedad. Así pues, la efectividad de un protocolo basado en el entrenamiento del ritmo beta1 ha sido probada en un estudio longitudinal de caso clínico. Una participante con síndrome de ansiedad completó 10 sesiones de NF, cuyo protocolo consistía en la potenciación del 
ritmo beta1 $(16-21 \mathrm{~Hz})$ e inhibición del ritmo theta (4-8 $\mathrm{Hz}$ ). Los niveles de ansiedad-estado y cortisol fueron registrados durante las 10 sesiones de acuerdo a un diseño pre/post. Se realizaron evaluaciones iniciales y finales de sintomatología ansiosa y rendimiento en atención sostenida. La evaluación final evidenció que los niveles de ansiedad volvieron a situarse en el rango normativo y la atención sostenida mostró importantes mejoras. Una prueba t para muestras relacionadas reveló un aumento significativo de la amplitud de betal entre sesiones sin cambios en ritmos no entrenados. Se evidenció una correlación significativa negativa entre la amplitud de beta1 y niveles de cortisol, sugiriendo que la actividad cerebral podría considerarse como posible marcador de ansiedad. La validación del protocolo se evaluó de acuerdo a criterios de independiencia, entrenabilidad e interpretabilidad. Se mostró la efectividad de un protocolo de NF sobre sintomatología ansiosa y atención sostenida, cuyo éxito residiría en el restablecimiento del arousal cortical óptimo capaz de inhibir la elevada actividad de la amígdala.

Palabras clave

Neurofeedback; ansiedad; cortisol; ritmo beta1; ritmo theta.

\section{Introduction}

Anxiety plays an adaptive role, as it empowers the individual in preparation for a probable dangerous situation. However, a state of excessively intense and prolonged anxiety alters the organism's homeostasis and leads to pathological states (Kumar, Rinwa, Kaur, \& Machawal, 2013), such as General Anxiety Disorder (GAD) or anxious symptomatology, which require intervention. Neurofeedback (NF) teaches to self-control brain activity according to operant principles with the aim of improving the mental state, whether or not there is a clinical condition (van Boxtel \& Gruzelier, 2014). The NF rationale implies: (a) proved associations between patterns of cortical activity and aspects of behavior; and (b) training to mirror said patterns to achieve changes in EEG and behavior (Vernon, 2005). An increasing number of well-controlled longitudinal NF studies have shown positive effects on a variety of neurological and psychological disorders, especially attention deficit hyperactivity disorder (ADHD) (Hammond, 2005; Moore, 2000; Moradi et al., 2011), although this technique still presents some issues of validation (Zoefel,
Huster \& Herrmann, 2011). The most frequently applied protocol is the enhancement of high frequency rhythms, such as alpha, sensorimotor rhythm (SMR) activity $(12-15 \mathrm{~Hz})$ or beta1 $(16-21 \mathrm{~Hz})$ activity, alone or in combination with the inhibition of theta activity $(4-7 \mathrm{~Hz})$ (Hammond, 2005; Moradi et al., 2011). The rationale behind the application of this type of protocol is based on previous findings: delta and theta oscillations have been shown to originate in the subcortical motivational/rewarding circuits, while the generation of alpha and beta oscillations are more closely related to cortical structures responsible for higher order mental functions, including cognitive and emotional regulation (Knyazev, 2007; Schutter \& Knyazev, 2012). In a healthy sample, the interchange between cortical and subcortical structures is characterised by a top-down communication (Goldin, McRae, Ramel, \& Gross, 2008; Van Honk \& Schutter, 2007) in which major amplitude of fast waves inhibit the amplitude of slow waves generated within deeper structures. Impulsive behaviour and emotionally driven behaviour have been associated with an excess of theta activity (Knyazev, 2007), which makes their bottom-up transmission disadvantageous and leads to dysfunctional emotional regulation (Putman, Van Peer, Maimari \& Van der Werff, 2010). The possibility of training specific EEG rhythms makes NF an excellent tool for treating anxiety disorders (Cheon, Koo \& Choi, 2016; Hammond, 2005; Moore, 2000; Moradi et al., 2011). The current study is innovative in that it measures a hormonal variable; cortisol. As an anxiety-observable product and a reliable biological marker for the assessment of hypothalamic-pituitary-adrenal (HPA) axis activity (Hellhammer, Wüst \& Kudielka, 2009), we were able to record several measures of salivary cortisol. Our results contribute to the limited data available regarding a relation between EEG bands, cortisol and behavior (Allen, Kennedy, Cryan, Dinan, \& Clarke, 2013; Dedovic et al., 2016; Hewig, Schlotz, Gerhards, Breitenstein, Lürken, \& Naumann, 2008).

This study was performed to determine, first of all, the reproducibility and practicality of 
an economic short-term NF intervention (10 sessions). Immediate and short-term effects of NF on absolute EEG band power, hormonal levels and clinical characteristics such as anxiety symptomatology and sustained attention were evaluated in a participant suffering from an anxiety syndrome. In line with the work of Zoefel et al. (2011), three main criteria were established to validate the NF protocol followed in this study: alterations of the desired bands, enhancement of beta1 and maintenance or a decrease of the theta band due to the training (trainability); a lack of change in untrained bands, for which we examined SMR (independence); a relation of the EEG changes with the affective/ cognitive variables of interest, which we expected to see manifested as a decrease in anxiety levels and improvement in sustained attention (interpretability). In addition, we expected to observe a relation (most likely inverse) between betal amplitude and cortisol levels throughout the NF training.

\section{Methods}

\section{Clinical case history}

The study's participant was a 33 year old Caucasian woman suffering from an anxiety syndrome, whose work had been compromised due to the anxiety caused by a legal/judicial situation regarding a traumatic event. She fulfilled the diagnostic criteria according to DSM-V (American Psychological Association, 2013): experiencing thoughts of worry (apprehensive expectation) difficult to control; persistent symptoms of excessive arousal and anxiety related to restlessness; sleep disturbance; and problems in concentration or working memory causing distress in social and professional areas. The participant did not receive any other treatment during the course of the experiment. The participant was treated in accordance with the Ethical Principles of Psychologists and Code of Conduct (American Psychological Association, 1992). All procedures were in accordance with the ethical standards of the institutional research committee and with the 1964 Helsinki declaration and its later amendments.

\section{Measures}

Psychological Tests

Cognitive assessment: The Toulouse-Piéron test (Toulouse \& Pieron, 1986) estimates perceptual speed and sustained attention. This instrument has a long history of administration and many derivations in experimental and clinical psychology. The task consists of a sheet containing 1.600 squares and the participant has to mark, during a 10-minute period, the squares that are in the same position as two given models. Mistakes in the subjects' performance of the task may be due to emotional and anxiety symptomatology, which is one of the reasons why this instrument was selected for our purpose of attentional assessment.

Anxiety assessment: The Spanish adaptation (Spielberger \& Gorsuch, 1982) of the StateTrait Anxiety Inventory (STAI) was used to assess situational and personal levels of anxiety. This tool is composed of two parts (each one with 20 items) and the total score in each part can vary from 20 to 80 . In addition, the Spanish version of the Inventory of Situations and Responses of Anxiety (ISRA) (Tobal \& Vindel, 1994) was administered. This task has a Situation-Response format to evaluate the frequency of several groups of anxiety behaviours: cognitive responses, physiologic responses and motor responses. It consists of 66 items, with 22 questions concerning each of the three aforementioned components plus a total trait of anxiety.

\section{EEG recording and Neurofeedback}

EEG signals were recorded, processed, filtered and represented using the BioGraph Infinity EEG Suite SA7950 Software. This software amplifies the amplitude of the frequency bands 
and the hardware Pro Comp 2 Infinity (Thought Technology Ltb; Montreal, Quebec) sends the signal to the computer. The EEG was used for both the recording and the feedback, which was sampled at $256 \mathrm{~Hz}$ samples/second and sent to the computer by the $A / D$ converter. The potential field was recorded through a monopolar electrode connection. In accordance with the international 10-20 system, an active scalp electrode was placed at C4 for theta/ betal training with the reference and ground electrode on the right and left earlobe, respectively. Impedance was maintained below $10 \mathrm{~K} \Omega$ and artefact rejection thresholds were set to suspend feedback when eye movements or other motor activity caused EEG fluctuations. The ongoing EEG at site, C4, was fast Fourier Transformed (FFT), band-pass filtered (from 0.1 to $60 \mathrm{~Hz}$ ) and notch filtered $(50 \mathrm{~Hz})$ in order to eliminate electrical interference and continuously measure the amplitude values for three bands - betal (16-21 Hz), SMR $(12-15 \mathrm{~Hz})$ and theta $(4-7 \mathrm{~Hz})$ amplitudes in microvolts, \#V, peak-to-peak, in accordance with the recommendations of previous reports (Gruzelier, Egner \& Vernon, 2006). A 4-min baseline was recorded before the NF to determine the threshold for reinforcement of each band. Feedback thresholds were automatically reset from block to block to maintain a constant level of reinforcement throughout the training session. Specifically, the software we employed obtains four means per second. Thus, regarding beta1 threshold, if the participant reaches $3 / 4$ means above the mean amplitude for the previous block then the reward is given and the threshold is raised 0.10 micro volts. In the case of the theta band, the participant must be $3 / 4$ means below the mean amplitude of the previous block. An audio-visual threshold-dependent protocol was used as reward stimuli feedback. When the EEG signals were within the thresholds, the patient obtained the reward, which consisted of continuation of the music and changing images. If the participant could not adjust to the thresholds, the audio would stop and the image would remain still.

\section{Biochemical analyses}

The participant provided two saliva samples in each of the 10 sessions by depositing $5 \mathrm{ml}$ of saliva in plastic vials during a period of no more than $5 \mathrm{~min}$. The samples were centrifuged at $3000 \mathrm{rpm}$ for $15 \mathrm{~min}$, resulting in a clear supernatant with low viscosity that was stored at $-20^{\circ} \mathrm{C}$ until analyses were performed. The samples were analyzed in the Central Research Unit of the Faculty of Medicine, University of Valencia (Spain) by a competitive solid phase radioimmunoassay (tube coated) using the commercial Cortisol RIA KIT Immunotech from Beckman Coulter Inc. (Prague, Czech Republic). All samples were measured in duplicate in the same trial. The within- and inter-assay variation coefficients were all below $4 \%$.

\section{Procedure}

After the subject completed an informed consent and medical questionnaire, an initial examination was carried out to establish a baseline for the following measures: Toulousse Test, ISRA, STAI-Trait and STAI-State. The NF sessions were then implemented twice a week until a total of 10 sessions had been completed. Each NF session was established as a pre-post design to obtain an estimation of anxiety (STAIState and STAI-Trait) and to collect a sample of salivary cortisol before and after receiving NF. The NF training consisted of three parts. After attaching the electrodes, the first EEG measure was taken as a baseline; during a 4-min period in an open-eye, resting state, without any feedback given, the participant was instructed to relax and focus on a symbol presented on a computer screen. This baseline was calculated following the instructions included with the software, with selected channels and the filtering of useful frequency bands. Subsequently, the NF session was initiated, consisting of 45 minutes of real feedback. The participant was instructed to relax and listen to the music and watch the computer screen so that the music would continue and the image would keep changing throughout the 
whole NF training session. At this point a further 4-min EEG recording was obtained, along with another cortisol sample and anxiety test. Later, the participant was cited one last time to assess alterations of the following measures: Toulousse Test, ISRA, STAI-Trait and STAI-State. The study was conducted in a quiet laboratory room.

\section{Statistical analysis}

First, we analyzed the amplitude of theta, SMR and betal bands for pre-training and posttraining across the 10 sessions. Normality of all dependent variables was confirmed with the Kolmogórov-Smirnov test (all $>$.05). A t-test for related samples was conducted for theta, SMR and betal amplitudes at pre and post NF training. Secondly, we performed two bivariate Spearman correlation analyses of pretraining and post-training brain band (beta1, theta and SMR) values and cortisol levels. Thirdly, to analyze the evolution of the STAIstate variable, the 10 training sessions were divided into two blocks to compare potential significant differences between the first 5 sessions and the second 5 sessions. Thus, a t-test of related samples was conducted for each of the two blocks of the pre-training phase, while the same strategy was implemented with the first 5 sessions and the last 5 sessions of the post-training phase. In addition, Spearman correlation analyses were conducted for STAI scores (STAI-State pretraining, STAI-State post-training and STAITrait variables) and time of evolution under the NF-training since initation, which provided information about the participant's anxiety throughout the intervention process. Regarding clinical outcomes, all scores obtained during the initial and final evaluation were transformed; a percentage was calculated for each subscale of each test taking into account the maximum possible score and the participant's actual score, in order to perform a better comparison between instruments. In addition, a percentage of improvement was calculated according to the following formula: [(pre-training - posttraining)/pre-training]*100 (Benvenuti, Buodo,
Leone \& Palomba, 2011). Raw scores were converted to $z$ scores [(raw score-mean score)/ standard deviation] and results were interpreted by comparing the patient's scores with normative data (Benvenuti et al., 2011). Once the $z$ score had been obtained and a standard deviation had been set as an outset (Sanz \& García-Vera, 2015), four ranges were determined to classify the scores: (a) performances out of normative range; (b) improved performance, but still out of normative range; (c) improved performance within a normative range; (d) performance within a normative range before and after training (Benvenuti et al., 2011). Finally, to test the effect size of the training on anxiety STAIState, we calculated the NonOverlap of All Pairs (NAP) index using the formula: NAP $=$ (№ NonOverlap pairs $)+(0.5 \times$ № ties $) / T o t a l$ № of pairs compared between baseline and treatment x 100 (Parker \& Vannest, 2009). All statistical analyses were performed using IBM SPSS 19 for Windows software.

\section{Results}

\section{Neurofeedback measures}

The analysis of betal amplitude revealed a significant main effect of Time, $t(9)=-3.99 ; p<$ 0.003; Cohen's $d=.90$, with an increase of mean amplitude from $12.13 \mu \mathrm{V}$ (SD 1.57) to 13.64 $\mu \mathrm{V}$ (SD 1.76). This implies that betal amplitude was significantly greater following training. With regard to theta amplitude, no main effect was found, $t(9)=1.07$; ns, although mean amplitude decreased after training from $9.47 \mu \mathrm{V}$ (SD .72) to $9.22 \mu \mathrm{V}$ (SD .52). The analysis of SMR mean amplitude did not reveal a significant main effect of Time either, $t(9)=-0.53$; ns, from $6.44 \mu \mathrm{V}$ (SD .74) to 6.57 (SD .62), which was expected taking into account that the SMR band was not trained in this case. The theta/beta1 ratio showed a significant effect of Time, $t(9)=3.17 ; p<0.01$; Cohen's $d=1.17$, with an increase from $1.28 \mu \mathrm{V}$ (SD .16) to $1.48 \mu \mathrm{V}$ (SD 0.18); this indicated a greater difference between the amplitude of the two EEG rhythms after training. A significant 
difference was not detected for the theta/SMR ratio, whose mean rose from .68 $\mu \mathrm{V}$ (SD .06) to $.71 \mu \mathrm{V}$ (SD .04). These results indicate that SMR and theta rhythms were not significantly affected by the betal uptraining.

\section{Cognitive and anxiety evaluation}

A clinical improvement was obtained progressively throughout the treatment period. A main effect was found regarding the progress of STAI State scores in the post-training episode, $t(4)=3.53 ; p=0.02$ with an effect of Cohen's $\mathrm{d}=1.49$, when the first five sessions were compared to the last five ones, from $M$ $=25.4(\mathrm{SD} 8.56)$ to $\mathrm{M}=15.8$ (SD 3.11). Inverse correlations were detected between STAI-State post-training and time of evolution $(r h o=-0.73 ; p<0.01)$, and between STAITrait and time of evolution (rho $=-0.75$; $p<0.01$ ), confirming that situational and trait anxiety decreased significantly as the NFtraining progressed. The clinical outcome with respect to all cognitive and anxiety scores is displayed in table 1 . The conversion of the raw scores to a percentage within each subscale and the percentage of improvement obtained show that every cognitive and anxiety variable improved considerably between the initial and final examination, particularly with respect to the ISRA-Motor scale and STAI-Trait scale, which showed the greatest progress. Moreover, after converting raw scores to $Z$ scores (see Table 1 ), we observed that most were out of normative range before the NF training, with the exception of the Toulouse score, ISRA-physiological score and STAI-State score, which were within normative range before training and remained so, despite a marked improvement, during the final examination. Upon completion of treatment, the participant's STAI-Trait score, ISRA-motor score, ISRA-cognitive score and ISRA-total score had improved and fell within normative range. Finally, we calculated the NAP index to confirm the therapeutic effect of the NF training on anxiety. According to Parker and Vannest's (2009) interpretation of the NAP index, the result of $70 \%$ indicates a medium effect of treatment when comparing the baseline anxiety score with scores throughout the course of the NF training; therefore, the result indicates a greater effect than chance. Significant correlations between these psychological measures and EEG values were not found (Table 1).

\section{TABLE 1}

All cognitive and anxiety scores of initial and final evaluations were converted into: (a) a percentage of each subescale of the test; (b) a percentage of improvement; and (c) z scores. To calculate the $z$ scores, the sample means and standard deviations of normative samples were used.

\begin{tabular}{|c|c|c|c|c|c|}
\hline & 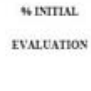 & 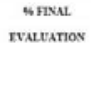 & S IMPROVEMENT & $\begin{array}{l}\text { Z SCORE } \\
\text { ESTRASCE } \\
\text { rVALATION }\end{array}$ & $\begin{array}{c}2 \text { SCORE } \\
\text { FEAL } \\
\text { Exacesto: }\end{array}$ \\
\hline Totzotse & 40 & 50.5 & 2625 & -0.696 & wher \\
\hline ISRA MOTOR & ss: & 20.16 & os. & L.S. & 0,000 \\
\hline ISRA COGSIIIVE & $49 s$ & sus & 12.36 & L.21\% & a ה \\
\hline ISRA nIYSIOLOCICAL & in & w.o & แ.1 & 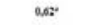 & s.6\% \\
\hline ISRLTOTAL TRAT & 3631 & 2013 & 225 & 1.25 & 0,000 \\
\hline STA-TRATr & 61.67 & 10.6 & nes & 1. & $\circ$ \\
\hline STMSTATR & 40 & nून & 2008 & 0.00 & wer \\
\hline
\end{tabular}

The $z$ scores were interpreted as follows: (a) Out of normative range. (b) Improved performance, but still out of normative range. (c) Improved performance within normative range. (d) Performance within normative range before the training and remained within this normative range after training. Source: own work

Biochemical analyses

Regarding cortisol, when the correlations between cortisol and brain activity were analyzed, an inverse correlation was found between the amplitude of betal and cortisol levels, rho $=-.69 ; p<0.02$, in the analyses executed on pre NF training values. No significant correlations were found on post NF training episode.

\section{Discussion}

The present study reports the outcomes of a NF protocol for treating anxious symptomatology. We aimed to investigate EEG changes across 
a 5-week NF training period using a wellestablished beta1/theta NF protocol to enhance beta1 without causing increases in theta rhythm. The criteria suggested by Zoefel et al. (2011) were followed (independence, trainability and interpretability) to evaluate the efficacy of the NF protocol administered. The outcome as a whole indicated positive results for our hypothesis. To assess the independence criterion, we observed if the EEG bands we had set out to train during the NF had in fact been trained and if untrained bands had remained unaltered. We expected to see a significant enhancement of the betal band and a decrease in the theta band with respect to baseline levels. Indeed, beta1 amplitude had increased significantly after the 5 weeks of training, without any significant changes in SMR rhythm being detected. The mean amplitude of the theta band decreased, though the change did not reach significance. The fact that no other rhythms were modified demonstrates the independence of the bands, as Egner and Gruzelier (2004) previously reported. Moreover, the results suggest good trainability. Indeed, it is reasonable to attribute the changes produced to the NF training, as the participant did not undergo any other type of intervention during the experiment.

Regarding interpretability, the results of the study are positive. NF training at C4 not only made a difference to EEG bands, but also had a considerable impact on all observable measures; functions that the participant had reported to be impaired previously. Regarding anxiety, both STAI trait and ISRA instruments showed significant reductions in their scores. In relation to ISRA outcomes, every score fell within the normative range after training, when the initial and final examinations were compared. Interestingly, the trait anxiety scale, in spite of its intrinsically stable nature, exhibited a marked decrease. This outcome is consistent with previous reports by Wu et al. (2014) and Moore (2000), who found that both state and trait anxiety scales were significantly reduced after intervention. These results encourage further research of this phenomenon to confirm that there exists a real long-term change in anxiety trait. Regarding evolution of the anxiety level throughout the NF training sessions, the evaluation of state anxiety showed a significant decrease during the last five sessions. Moreover, the inverse correlations detected between STAI values - both situational and trait anxiety - and the evolution of time under the NF-training support a beneficial effect of NF on anxiety symptomatology; thus, the more sessions the participant underwent, the lower her anxiety levels were. This result is consistent with the study of Moradi et al. (2011), in which major progress in alpha and beta amplitude was obtained during the last part of the intervention. Additionally, the NAP index, usually employed as the most statically trustworthy index for the analysis of single case studies (Parker \& Vannest, 2009), indicated a middle size effect of the NF training on anxiety. Although the size effect obtained for this study is not one of $100 \%$, it confirms a treatment effect undeniably greater than chance. Indeed, several reports point to an association between a lack of attention and low beta or alpha activity (Gruzelier et al., 2006; Moradi et al., 2011; Vasquez, Gadea, Garijo, Aliño, \& Salvador, 2015). Moradi et al. (2011) found that patients suffering chronic anxiety, and who exhibited abnormally low levels of beta and alpha activity and abnormally high levels of beta2, improved significantly in anxiety and attention after NF. The results of the current study suggest similar effects. Following beta1/ theta NF training, sustained attention showed an increase of $26.25 \%$, which means that the patient improved in perceptual speed without increasing the number of mistakes or omissions. As our results show, we did not find significant correlations between EEG modifications and psychological measures that would confirm the interpretability criteria. In spite of this, the results showing changes in EEG bands, anxiety and sustained attention, as a whole, provide evidence of important modifications in the desired direction. It would be of interest for future studies to employ larger samples to allow a better statistical study of the effects.

The results of this study are consistent with the biological basis of the emotional regulation 
theory mentioned in the Introduction (Knyazev, 2007; Goldin et al., 2008) and justify the applicability and effectiveness of a beta1/theta protocol not only for ADD/ADHD population, as literature suggests, but for treatment of anxiety as well. When a control sample was included in other studies (Knyazev, 2007; Schutter \& Knyazev, 2012; van Honk \& Schutter, 2007), it was characterized by an inhibition-regulation mechanism that the cortex exerts on deeper brain structures. As Brennan and Arnsten (2008) observed, people diagnosed with ADD/ADHD present an increased power of slow waves with respect to control samples. Thus, Lubar (1991) proposed that training the betal band may serve to raise cortical excitation in under-aroused samples. Moreover, patients with mood and anxiety disorders tend to show a hypoactive rostral cingulate in tasks involving emotional processing or distraction when they experience greater interference from emotional distracters (Etkin, Egner, Peraza, Kandel \& Hirsch, 2006; Hull, 2002). Etkin et al. (2006) suggested that elevated amygdalar activity and exaggerated behavioural interference may be due to deficient amygdalar inhibition by the rostral cingulate, leading to an inability to deal with emotional conflict. In this context, Cheon, Koo and Choi (2016) administered a combination of beta and alpha/theta NF training to patients with major depressive disorder and comorbility with anxiety symptoms. They found that NF improved depressive symptoms significantly, as well as anxiety symptoms and clinical illness severity. Additionally, in previous studies in our laboratory (Vasquez et al., 2015), we have found that enhancement of the beta1 band and inhibition of theta produced significant positive changes in executive performance and reduced negative mood. Thus, low power of fast waves may reflect reduced frontal cortical functions and, in turn, an impaired inhibitory function and emotional instability (Putman et al., 2010), which is known to be affected in anxiety disorder (Goldin et al., 2008). Although the present results should be taken with caution due to the nature of the study, they are consistent with literature sustaining the effectiveness of a fast wave NF protocol.
In addition to the psychological measures and EEG changes, the inverse association found in the current study between betal amplitude and cortisol levels provides further evidence of the relation between this hormone and cortical activation (Allen et al., 2013; Dedovic et al., 2016; Hewig et al., 2008). Indeed, lower amplitude of betal was associated with higher cortisol levels. This fact could in turn be related to higher levels of anxiety, though this relation could not be confirmed in this study, possibly due to the small sample. All in all, our results endorse previous existing evidence (Hewig et al., 2008) suggesting that an improvement at a neuroendocrine, behavioral and executive level could be obtained through fast wave NF training. We suspect that this training exerts a regulatory effect on overactive subcortical areas linked to anxiety and emotional instability. However, this mechanism should be explored more extensively in future studies by measuring cortisol levels in hair, which would provide information regarding cortisol concentration and the state of the patient during the last three months (Karlén, Ludvigsson, Frostell,Theodorsson \& Faresjö, 2011; Stalder et al., 2014).

To our knowledge, this is the first longitudinal study to assess NF, hormonal, cognitive and emotional variables according to the criteria suggested by Zoefel et al. (2011) to assure the validation of a NF protocol. Our results suggest that the training of these specific bands can be effective in an anxious patient and confirm the inhibitory function theory. Moreover, the relation observed between beta1 band and cortisol levels suggest that brain activity may be considered a marker of anxiety in the near future. More research is needed to investigate the underlying mechanism of action so that this protocol can eventually be consolidated as a safe and effective intervention.

\section{References}

Allen, A.P, Kennedy, P.J., Cryan, J.F., Dinan, T.G., \& Clarke, G. (2014). Biological and psychological markers of stress in 
humans: Focus on the Trier Social Stress Test. Neuroscience \&ु Biobehavioral Reviews, 38, 94-124. doi: 10.1016/ j.neubiorev.2013.11.005

American Psychological Association (1992). Ethical principles of psychologists and code of conduct. American Psychologist, 47, 1597-1611.

American Psychiatric Association (2013). Diagnostic and Statistical Manual of Mental Disorders, Fifth Edition. Arlington, VA, American Psychiatric Association.

Benvenuti, S. M., Buodo, G., Leone, V., \& Palomba, D. (2011). Neurofeedback training for Tourette Syndrome: An uncontrolled single case study. Applied Psychophysiology and Biofeedback, 36(4), 281-288.

Brennan, A. R. \& Arnsten, A. F. (2008). Neuronal mechanisms underlying attention deficit hyperactivity disorder: The influence of arousal on prefrontal cortical function. Annals of the New York Academy of Sciences, 1129, 236-245.

Cheon, E. J., Koo, B. H., \& Choi, J. H. (2016). The efficacy of neurofeedback in patients with major depressive disorder: An open labelled prospective study. Applied Psychophysiology and Biofeedback, [Epub ahead of print] doi: 10.1007/ s10484-015-9315-8.

Dedovic, K., Giebl, S., Duchesne, A., Lue, S. D., Andrews, J. ... \& Pruessner, J. C. (2016). Psychological, endocrine and neural correlates of attentional bias in subclinical depression, Anxiety, Stress, EF Coping, 29 (5), 479-496. Doi: $10.1080 / 10615806.2015 .1101457$

Egner, T., \& Gruzelier, J. H. (2004). EEG biofeedback of low beta band components: frequency specific effects on variables of attention and event-related brain potentials. Clinical Neurophysiology, 115(1), 131-9.

Elkins, G., Rajab, M. H., \& Marcus, J. (2005). Complementary and alternative medicine use by psychiatric inpatients. Psychological Reports, 96, 163-166.
Etkin, A., Egner, T., Peraza, D.M., Kandel, E.R., \& Hirsch, J. (2006). Resolving emotional conflict: A role for the rostral anterior cingulate cortex in modulating activity in the amygdale. Neuron, 51 (6), 871-882.

Goldin, P.R., McRae, K., Ramel, W., \& Gross, J.J. (2008). The neural bases of emotion regulation: reappraisal and suppression of negative emotion. Biological Psychiatry, 63(3), 577-586.

Gruzelier, J.H., Egner, T., \& Vernon, D. (2006). Validating the efficacy of neurofeedback for optimising performance. Progress in Brain Research, 159, 421-431.

Hammond D. (2005). Neurofeedback with anxiety and affective disorders. Child E Adolescent Psychiatric Clinics of North America, 14(1), 105-123.

Hellhammer, D. H., Wüst, S., \& Kudielka, B. G. (2009). Salivary cortisol as a biomarker in stress research. Psychoneuroendocrinology, 34(2), 163-171.

Hewig, J., Schlotz, W., Gerhards, F., Breitenstein, C., Lürken, A., \& Naumann, E. (2008). Associations of the cortisol awakening response (CAR) with cortical activation asymmetry during the course of an exam stress period. Psychoneuroendocrinology, $33(1), 83-91$.

Hull, A.M. (2002). Neuroimaging findings in post-traumatic stress disorder. Systematic review. The British Journal of Psychiatry, 181, 102-110.

Karlén, J., Ludvigsson, J., Frostell, A., Theodorsson, E., \& Faresjö, T. (2011). Cortisol in hair measured in young adults - a biomarker of major life stressors? BMC Clinical Pathology, 11, 12.

Knyazev, G. G. (2007). Motivation, emotion, and their inhibitory control mirrored in brain oscillations. Neuroscience $\mathbb{E}$ Biobehavioral Reviews, 31(3), 377-395.

Kumar, A., Rinwa, P., Kaur, G., \& Machawal, L. (2013). Stress: Neurobiology, consequences and management. Journal of Pharmacy and Bioallied Sciences, 5(2), 91-97.

Lubar, J.F. (1991). Discourse on the development of EEG diagnostics and biofeedback for 
attention-deficit/hyperactivity disorders. Biofeedback and Self-Regulation Journal, 16(3), 201-225.

MacNamara, A., \& Proudfit, G. H. (2014). Cognitive load and emotional processing in generalized anxiety disorder: electrocortical evidence for increased distractibility. Journal of Abnormal Psychology, 123(3), 557-565.

Moore, N.C. (2000). A review of EEG Biofeedback treatment of anxiety disorders. Clinical electroencephalography, 31 (1), 1- 6.

Moradi, A., Pouladi, F., Pishva, N., Rezaei, B., Torshabi, M., \& Mehrjerdi, Z. (2011). Treatment of Anxiety Disorder with Neurofeedback: case study. Procedia-Social and Behavioral Sciences 30, 103-107.

Parker, R. I. \& Vannest, K. J. (2009). An improved effect size for single case research: NonOverlap of All Pairs (NAP). Behavior Therapy, 40, 357-367.

Putman, P., van Peer, J., Maimari, I., \& van der Werff, S. (2010). EEG theta/ beta ratio in relation to fear-modulated response-inhibition, attentional control, and affective traits. Biological Psychology, 83(2), 73-78.

Sanz, J. \& García-Vera, M.P. (2015). Técnicas para el análisis de diseños de caso único en la práctica clínica: ejemplos de aplicación en el tratamiento de víctimas de atentados terroristas. Clínica y Salud, 26, 167-180.

Schutter, D. J. L. G., \& Knyazev, G. G. (2012). Cross-frequency coupling of brain oscillations in studying motivation and emotion. Motivation and Emotion Journal, 36(1), 46-54.

Spielberger, C.D., Gorsuch, R.L., \& Lushene, R.E. (1982) STAI: Cuestionario de Ansiedad Estado-Rasgo. Manual. Madrid: TEA Ediciones, S.A.

Stalder, T., Tietze, A., Steudte, S., Alexander, N., Dettenborn, L., \& Kirschbaum, C. (2014). Elevated hair cortisol levels in chronically stressed dementia caregivers. Psychoneuroendocrinology, 47, 26-30.
Tobal, J. J. M., \& Vindel, A. R. C. (1994) ISRA: Inventario de Situaciones y Respuestas de Ansiedad: Manual. Madrid: TEA Ediciones.

Toulouse, E., \& Pieron, H. (1986). ToulousePieron: Prueba perceptiva y de atención: Manual. Madrid: TEA Ediciones.

Van Boxtel, G. J. M., \& Gruzelier, J. H. (2014). Neurofeedback: Introduction to the special issue. Biological Psychology, 95, 1-3. Doi: 10.1016/j.biopsycho.2013.11.011

Van Honk, J., \& Schutter, D. J. L. G. (2007). Testosterone reduces conscious detection of signals serving social correction: Implications for antisocial behaviour. Psychological Science, 18(8), 663-667.

Vasquez, M., Gadea, M., Garijo, E., Aliño, M., \& Salvador, A. (2015). Efectos del entrenamiento asistido con neurofeedback sobre EEG, los procesos de función ejecutiva y el afecto en una muestra de población normal. Anales de Psicología, 31 (1), 317-323. http://dx.doi.org/10.6018/a nalesps.31.1.167241

Vernon, D. (2005). Can Neurofeedback Training Enhance Performance? An Evaluation of the Evidence with Implications for Future Research. Applied Psychophysiology and Biofeedback, 30 (4), 347-364.

Wu, J., Cui, Y., Yang, Y., Kang, M., Jung, S., ... \& Eun, S. (2014). Modulatory effects of aromatherapy massage intervention on electroencephalogram, psychological assessments, salivary cortisol and plasma brain-derived neurotrophic factor. Complementary Therapies in Medicine, 22, 456-462.

Zoefel, B., Huster, R. J., \& Herrmann, C. S. (2011). Neurofeedback training of the upper alpha frequency band in EEG improves cognitive performance. Neuroimage, 54(2), 1427-1431.

\section{Notes}

* Research Article 